\title{
Impact of Catchment Area Activities on Water Quality in Small Retention Reservoirs
}

\author{
Katarzyna Oszczapińska ${ }^{1,}$, Iwona Skoczko ${ }^{1}$, and Joanna Szczykowska ${ }^{1}$ \\ ${ }^{1}$ Department of Technology in Engineering and Environmental Protection, Bialystok University of \\ Technology, Wiejska St. 45E, 15-351 Bialystok
}

\begin{abstract}
The aim of the study was to evaluate catchment area impact on small water reservoirs condition in Podlasie. The researches were conducted in two different catchment areas. Topiło reservoir, located in Podlasie area in the south-east of Białowieża Forest, has typical sylvan catchment. Second reservoir, Dojlidy, is located also in Podlasie, in the south-east of Białystok as a part of Dojlidy Ponds. In contrast to Topiło, Dojlidy has agricultural catchment. Water samples collected from five sites along each reservoir were analysed for the presence of total nitrogen and phosphorus, chlorophyll "a", reaction, turbidity and conductivity. Researches took place in spring, summer and autumn 2013 (Topiło Lake) and 2014/2015 (Dojlidy). The lowest trophic state was observed in autumn and the highest in summer. Because of the high loads of phosphorus received by the reservoirs, this element did not limit primary production. Calculated TSI values based on total phosphorus were always markedly higher than calculated on chlorophyll-a and total nitrogen. Both reservoirs demonstrated TSI indexes specific to hypertrophic lakes due to large amount of total phosphorus.
\end{abstract}

\section{Introduction}

There is close relationship between surface water quality and its catchment area character. Chemical substances scouring, conditioned on the type of land use, geological substratum structure, kind of soil and climate conditions, has significant impact on chemical water components changes [1].

Right functioning of small retention reservoir depends on water quality. Objects located in agricultural catchment are particularly exposed to fertilizers, pesticides and organic matter eluted from the soil. Small and medium reservoirs depth aids eutrophication. Often it comes to supply watercourse pollution [2].

The most common biogenic sources in surface waters are, mentioned earlier, fertilizers used in agricultural catchments. Their influent on soils in catchment basin was tested by Czyżyk and Rajmund (2013), who after six-year studies proved that more nitrogen has permeated to the soil from mineral than natural fertilizers [3].

On the other hand, sylvan catchments exhibit smaller biogenic runoff thanks to forest cover absorption abilities [4].

\footnotetext{
* Corresponding author: k.oszczapinska@doktoranci.pb.edu.pl
} 


\section{Methods}

Studies were conducted in two different catchment areas. Topiło reservoir, located in Podlasie area in the south-east of Białowieża Forest, has typical sylvan catchment. It was built in 1932-1933 as a wooden logs store, but in 1975 tank was excluded from this type of use. Topiło Lake catchment area with Perebel River involves Białowieża Forest and Hajnówka urban area. Topiło location bears on industrial and municipal pollution character. Reservoir has 21.1 ha water surface and only $1 \mathrm{~m}$ of average depth [5].

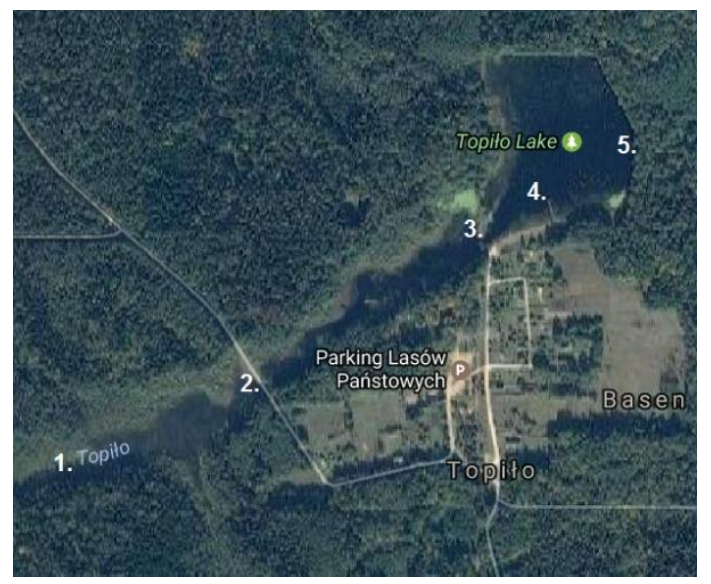

Fig. 1. Location of control points within Topiło Lake [6].

Second reservoir, Dojlidy, is located also in Podlasie area, in the south-east of Białystok as a part of Dojlidy Ponds. In contrast to Topiło Dojlidy, with 34 ha and $1.75 \mathrm{~m}$ average depth, has agricultural catchment. It was built in 1962 by damming Biała River. The research object is being used as a swimming pool, but in the period of the study it was excluded from this type of use because of modification works made by the Water Sports Centre "Dojlidy". It is also a place for extensive, non-commercial fish farming. In addition, the reservoir has the task of regulating water surface runoff [7].

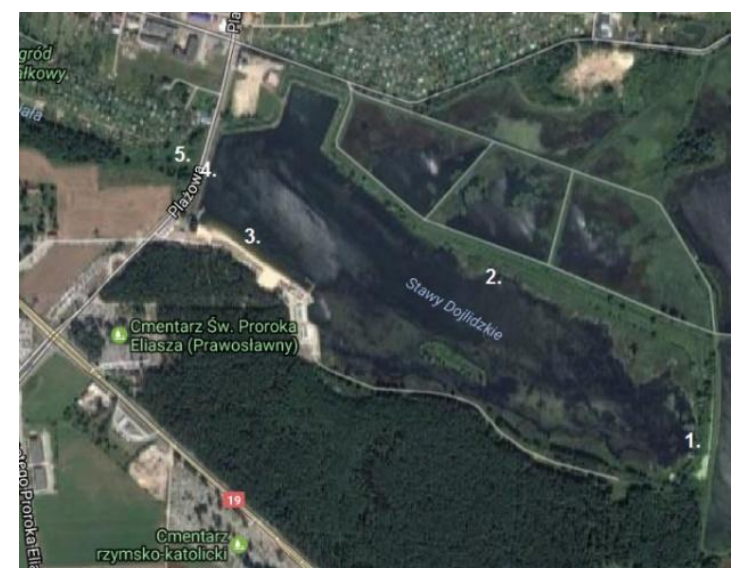

Fig. 2. Location of control points within Dojlidy [6]. 
Along each reservoir five sites were appointed. The sampling points were distributed in specific areas within Topiło and Dojlidy to allow the observation of changes dynamics in the ecological status of the objects. Within Topiło Lake points were located consecutively: first near the Perebel River flow into the reservoir, second next to the Topiło-Łozice bridge, third beside water dam with railroad, fourth by the side of wooden dock and fifth on the Perebel River, behind the reservoir (Fig. 1). The first point in Dojlidy was located near the Biala River flow into the reservoir. The second point included central, coastal part of the object. The third point was located nearby the bridge on the beach. The fourth point was the place of water outflow over the front dam, while the fifth point was on the Biala River, behind the reservoir (Fig. 2). Researches took place in spring, summer and autumn 2013 (Topiło Lake) and 2014/2015 (Dojlidy). Each time of the year the samples were collected twice, from a depth of about $0.4 \mathrm{~m}$. They were analysed for the presence of the following indicators: total nitrogen, total phosphorus, chlorophyll "a", reaction, turbidity, and conductivity. Different parameters were determined by a UV-VIS spectrometry according to the following methods [8-10]:

- total nitrogen - Total Kjeldahl Nessler Method, HACH® Company,

- total phosphorus - PhosVer ${ }^{\circledR} 3$ with Acid Persulfate Digestion Method, HACH® Company,

- chlorophyll "a" - spectrophotometric, monochromatic method with feopigments alfa correction,

- reaction - electrometric method, CP-401 Elmetron pH-meter,

- turbidity - nephelometry method, HACH® Company,

- conductivity - electrometric method, Slandi CM 204 conductometer.

\section{Results and discussion}

Concentrations of total nitrogen and total phosphorus in water were characterized by remarkable variability. Seasonal changes in these indicators of water quality during research period were recorded. The lowest concentrations of total nitrogen were observed in summer and autumn, while the highest in spring (Table 1). According to Environment Minister Regulation from 21 July 2016 [11] total nitrogen concentration for I and II water quality class in reservoir should not be higher than $2 \mathrm{mg} / \mathrm{dm}^{3}$. Topiło and Dojlidy reservoirs, with relatively large total nitrogen amount, cannot be classified to first and second water quality class.

Table 1. Average, minimum-maximum total nitrogen amount in Topiło and Dojlidy.

\begin{tabular}{|c|c|c|c|c|c|c|c|c|c|c|}
\hline $\begin{array}{c}\text { Total } \\
\text { nitrogen }\end{array}$ & \multicolumn{5}{|c|}{ Topiło } & \multicolumn{5}{|c|}{ Dojlidy } \\
\hline $\begin{array}{c}\text { Control } \\
\text { point }\end{array}$ & 1. & 2. & 3. & 4. & 5. & 1. & 2. & 3. & 4. & 5. \\
\hline & 6.40 & 4.29 & 5.08 & 3.94 & 3.58 & 6.13 & 1.25 & 2.63 & 0.63 & 2.13 \\
\hline Spring & $\begin{array}{l}\overline{5,96}- \\
7,27\end{array}$ & $\begin{array}{c}\overline{3,84}- \\
4,78\end{array}$ & $\begin{array}{c}\overline{4,59-} \\
5,27\end{array}$ & $\begin{array}{l}3,52- \\
4,42\end{array}$ & $\begin{array}{l}2,65- \\
4,20\end{array}$ & $\begin{array}{c}\overline{4,09}- \\
6,89\end{array}$ & $\begin{array}{l}\overline{0,58-} \\
2,02\end{array}$ & $\begin{array}{l}2,45- \\
2,89\end{array}$ & $\begin{array}{c}\overline{0,21-} \\
0,98\end{array}$ & $\begin{array}{l}\overline{0,87-} \\
2,54\end{array}$ \\
\hline & 2.09 & 2.08 & 2.29 & 1.67 & 1.88 & 3.25 & 1.38 & 1.88 & $\underline{3.88}$ & 2.88 \\
\hline Summer & $\begin{array}{l}\overline{0,65-} \\
3,10\end{array}$ & $\begin{array}{l}0,83- \\
2,95\end{array}$ & $\begin{array}{l}1,25- \\
3,00\end{array}$ & $\begin{array}{l}0,68- \\
2,92\end{array}$ & $\begin{array}{l}0,87- \\
3,21\end{array}$ & $\begin{array}{l}3,11- \\
3,85\end{array}$ & $\begin{array}{l}1,01- \\
1,55\end{array}$ & $\begin{array}{l}\overline{1,12-} \\
2,05\end{array}$ & $\begin{array}{l}2,22- \\
4,10\end{array}$ & $\begin{array}{c}2,55- \\
3,06\end{array}$ \\
\hline Autumn & $\begin{array}{l}\frac{1.04}{0,50-} \\
1,16\end{array}$ & $\begin{array}{r}\frac{1.21}{0,74-} \\
1,35\end{array}$ & $\begin{array}{l}\frac{1.79}{0,85-} \\
2,02\end{array}$ & $\begin{array}{c}\underline{0.67} \\
0,42- \\
0,89\end{array}$ & $\begin{array}{c}\underline{0.50} \\
0,38- \\
0,74\end{array}$ & $\begin{array}{c}\frac{0.38}{0,11-} \\
0,86\end{array}$ & $\begin{array}{l}\frac{1.50}{1,01-} \\
1,87\end{array}$ & $\begin{array}{c}\frac{0.88}{0,52-} \\
1,02\end{array}$ & $\begin{array}{c}\frac{0.75}{0,33-} \\
1,16\end{array}$ & $\begin{array}{r}\underline{0.63} \\
0,21- \\
0,95\end{array}$ \\
\hline
\end{tabular}


Highest total phosphorus concentrations in both reservoirs were notified in spring and autumn. Total phosphorus amount was greater in Topiło Lake, like total nitrogen concentration (Table 2). The two studied objects were marked by large total phosphorus amount, which was much higher than pollution limit for first and second water quality class (accordingly $\leq 0.045 \mathrm{mgP} / \mathrm{dm}^{3}$ and $\leq 0.060 \mathrm{mgP} / \mathrm{dm}^{3}$ ) [11].

Table 2. Average, minimum-maximum total phosphorus amount in Topiło and Dojlidy.

\begin{tabular}{|c|c|c|c|c|c|c|c|c|c|c|}
\hline $\begin{array}{c}\text { Total } \\
\text { phosphorus }\end{array}$ & & & Topiło & & & & & Dojlidy & & \\
\hline $\begin{array}{c}\text { Control } \\
\text { point }\end{array}$ & 1. & 2. & 3. & 4. & 5. & 1. & 2. & 3. & 4. & 5. \\
\hline & 2.24 & 1.95 & $\underline{1.85}$ & 1.33 & 1.47 & 2.17 & $\underline{2.30}$ & $\underline{1.95}$ & $\underline{1.59}$ & 1.88 \\
\hline Spring & $\begin{array}{l}\overline{1,76-} \\
2,84\end{array}$ & $\begin{array}{l}1,62- \\
2,35\end{array}$ & $\begin{array}{l}1,56- \\
2,19\end{array}$ & $\begin{array}{l}0,74- \\
2,02\end{array}$ & $\begin{array}{l}\overline{0,93-} \\
2,10\end{array}$ & $\begin{array}{l}1,52- \\
2,29\end{array}$ & $\begin{array}{l}1,68- \\
2,57\end{array}$ & $\begin{array}{l}1,85- \\
2,32\end{array}$ & $\begin{array}{l}1,02- \\
1,70\end{array}$ & $\begin{array}{l}\overline{1,60-} \\
2,04\end{array}$ \\
\hline & 1.09 & $\underline{0.90}$ & $\underline{1.53}$ & 1.22 & 0.98 & $\underline{0.58}$ & $\underline{0.55}$ & $\underline{0.42}$ & $\underline{0.63}$ & 0.48 \\
\hline Summer & $\begin{array}{l}\overline{0,90-} \\
1,24\end{array}$ & $\begin{array}{l}\overline{0,75-} \\
1,10\end{array}$ & $\begin{array}{l}\overline{0,62-} \\
2,20\end{array}$ & $\begin{array}{c}\overline{0,54-} \\
1,95\end{array}$ & $\begin{array}{l}\overline{0,31-} \\
1,33\end{array}$ & $\begin{array}{c}\overline{0,23-} \\
0,66\end{array}$ & $\begin{array}{c}\overline{0,39-} \\
0,98\end{array}$ & $\begin{array}{c}\overline{0,30-} \\
0,49\end{array}$ & $\begin{array}{c}\overline{0,58-} \\
0,71\end{array}$ & $\begin{array}{c}\overline{0,36}- \\
0,52\end{array}$ \\
\hline & 0.68 & 1.31 & 2.44 & 2.66 & 0.30 & 2.30 & 1.14 & $\underline{0.93}$ & 1,80 & $\underline{1.00}$ \\
\hline Autumn & $\begin{array}{c}0,55- \\
0,92\end{array}$ & $\begin{array}{l}1,06- \\
1,62\end{array}$ & $\begin{array}{c}2,21- \\
2,96\end{array}$ & $\begin{array}{c}2,07- \\
2,81\end{array}$ & $\begin{array}{r}0,12- \\
1,04\end{array}$ & $\begin{array}{c}2,16- \\
2,39\end{array}$ & $\begin{array}{c}0,98- \\
1,22\end{array}$ & $\begin{array}{c}0,86- \\
1,08 \\
\end{array}$ & $\begin{array}{l}1,23- \\
2,14\end{array}$ & $\begin{array}{c}0,68- \\
1,19\end{array}$ \\
\hline
\end{tabular}

Seasonal differences in nutrients concentrations in water of the reservoirs relate to plant's vegetation [12]. Factor that caused high concentrations of both nitrogen and phosphorus could be probably sediments covering bottom of the reservoirs, which in Topiło came from wooden logs stored in the past. Because of lack of regular sediment removal thick layer in Dojlidy have agglomerated [13].

The content of chlorophyll "a" in waters body of both studied reservoirs was also subject to seasonal changes. Largely higher concentrations were noticed in Topiło with maximum $34.94 \mu \mathrm{g} / \mathrm{dm}^{3}$ in summer, while the highest average chlorophyll "a" amount in Dojlidy reached $16.02 \mu \mathrm{g} / \mathrm{dm}^{3}$. Chlorophyll "a" concentration increase was observed while the intensive autotrophic microbes growth. It slightly decreased in autumn, as the vegetation season was ending (Table 3).

A beneficial condition for phytoplankton development is low average depth below $2 \mathrm{~m}$ and connected with that biogenic compound dilution in smaller water volume [14].

Table 3. Average, minimum-maximum chlorophyll "a" amount in Topiło and Dojlidy.

\begin{tabular}{|c|c|c|c|c|c|c|c|c|c|c|}
\hline $\begin{array}{c}\text { Chlorophyll } \\
\left.\text { "a" [g/dm }{ }^{3}\right]\end{array}$ & \multicolumn{9}{|c|}{ Topiło } & \multicolumn{5}{c|}{ Dojlidy } \\
\hline $\begin{array}{c}\text { Control } \\
\text { point }\end{array}$ & 1. & 2. & 3. & 4. & 5. & 1. & 2. & 3. & 4. & 5. \\
\hline \multirow{3}{*}{ Spring } & $\frac{13.36}{4,01}$ & $\frac{19.22}{5,30}$ & $\frac{24.90}{6,68}$ & $\frac{17.86}{5,32}$ & $\frac{15.69}{4,26}$ & $\frac{14.69}{5,85}$ & $\frac{11.35}{3,70}$ & $\frac{16.02}{4,69}$ & $\frac{2.67}{2,01}$ & $\frac{4.01}{3,94}$ \\
& - & - & - & - & - & - & - & - & - & - \\
& 19,04 & 33,37 & 41,04 & 29,70 & 27,37 & 16,04 & 15,37 & 18,22 & 3,34 & 4,69 \\
\hline & $\underline{34.12}$ & $\underline{34.94}$ & $\underline{32.58}$ & $\underline{31.47}$ & $\underline{24.52}$ & $\underline{4.01}$ & $\underline{12.28}$ & $\underline{7.12}$ & $\underline{6.23}$ & $\underline{2.00}$ \\
Summer & 3,75 & 34,13 & 32,52 & 30,63 & 17,80 & 2,01 & 4,52 & 6,94 & $-4,01$ & 0,67 \\
& - & - & - & - & - & - & - & - & - & - \\
& 36,04 & 35,50 & 33,04 & 32,24 & 25,97 & 5,67 & 13,6 & 8,34 & 7,58 & 3,56 \\
\hline & 14.04 & $\underline{12.03}$ & $\underline{15.34}$ & $\underline{14.03}$ & $\underline{6.01}$ & $\underline{1.78}$ & $\underline{5.34}$ & $\underline{3.56}$ & $\underline{2.67}$ & $\underline{0.89}$ \\
Autumn & 5,68 & 5,52 & 6,04 & 5,97 & 4,01 & 0,37 & 2,01 & 1,67 & 0,97 & 0,30 \\
& - & - & - & - & - & - & - & - & - & - \\
& 23,04 & 19,37 & 21,13 & 23,37 & 7,68 & 2,67 & 7,90 & 5,52 & 5,01 & 1,12 \\
\hline
\end{tabular}


Summary statistics for turbidity, $\mathrm{pH}$, and conductivity (Table 2) did not indicate impaired water quality in any of the reservoirs. Measured water turbidity was never greater than 37 NTU in Topiło, and 29 in Dolidy, measured pH values were never less than 6,88 in Topiło, and 7.25 in Dojlidy. Water conductivity was never greater than $321 \mu \mathrm{S} / \mathrm{cm}$ in Topiło, and 518 in Dojlidy.

Much higher water turbidity was measured in Topiło, especially during summer and autumn researches. Dojlidy in turn was characterized by lower turbidity (Table 4).

Table 4. Topiło and Dojlidy average, minimum-maximum water turbidity.

\begin{tabular}{|c|c|c|c|c|c|c|c|c|c|c|}
\hline \multirow{2}{*}{$\begin{array}{c}\text { Turbidity } \\
{[\text { NTU] }}\end{array}$} & \multicolumn{5}{|c|}{ Topiło } & \multicolumn{5}{|c|}{ Dojlidy } \\
\hline & 1. & 2. & 3. & 4. & 5. & 1. & 2. & 3. & 4. & 5. \\
\hline \multirow{4}{*}{ Spring } & $\underline{5}$ & $\underline{6}$ & $\underline{3,5}$ & $\underline{3,5}$ & $\underline{2}$ & $\underline{10}$ & 1 & $\underline{9}$ & 29 & $\underline{13}$ \\
\hline & 3 & 5 & $\overline{2}$ & $\overline{1,5}$ & $\overline{1,4}$ & 6 & 0,5 & $\overline{7}$ & $\overline{23}$ & 11 \\
\hline & - & - & - & - & - & - & - & - & - & - \\
\hline & 8 & 6,6 & 5,7 & 5 & 3 & 12 & 2 & 10 & 32 & 14 \\
\hline \multirow{4}{*}{ Summer } & 31 & 24 & $\underline{12}$ & 7 & 20 & $\underline{9}$ & $\underline{3}$ & 1 & $\underline{5}$ & 1 \\
\hline & 25 & 20 & $\overline{9}$ & 5 & $\overline{15}$ & 8 & 1 & 0,5 & 3 & 0,5 \\
\hline & - & - & - & - & - & - & - & - & - & - \\
\hline & 39 & 26 & 21 & 11 & 24 & 11 & 4 & 2 & 6 & 3 \\
\hline \multirow{4}{*}{ Autumn } & 23 & 37 & 29 & 21 & 20 & 13 & 4 & $\underline{3}$ & 22 & 25 \\
\hline & 17 & 25 & 22 & 17 & 7 & 10 & 3 & 1 & 17 & 21 \\
\hline & - & - & - & - & - & - & - & - & - & - \\
\hline & 24 & 41 & 33 & 22 & 24 & 14 & 7 & 4 & 23 & 28 \\
\hline
\end{tabular}

Summer researches demonstrated similar level in both objects. On the other hand, Topiło reservoir was marked by slightly lower water reaction in spring and autumn than Dojlidy (Table 5).

Table 5. Topiło and Dojlidy water reaction (median, minimum-maximum).

\begin{tabular}{|c|c|c|c|c|c|c|c|c|c|c|}
\hline $\begin{array}{c}\text { Reaction } \\
{[\mathrm{pH}]}\end{array}$ & \multicolumn{5}{|c|}{ Topiło } & \multicolumn{5}{|c|}{ Dojlidy } \\
\hline $\begin{array}{c}\text { Control } \\
\text { point }\end{array}$ & 1. & 2. & 3. & 4. & 5. & 1. & 2. & 3. & 4. & 5. \\
\hline Spring & $\begin{array}{l}\frac{7.02}{6,94-} \\
7,60\end{array}$ & $\begin{array}{l}\frac{6.88}{6,62-} \\
7,30\end{array}$ & $\begin{array}{l}\underline{6.97} \\
6,85- \\
7,33\end{array}$ & $\begin{array}{l}\frac{7.03}{6,94-} \\
7,28\end{array}$ & $\begin{array}{l}\frac{6.95}{6,87-} \\
7,40\end{array}$ & $\begin{array}{l}\frac{7.25}{7,02-} \\
7,41\end{array}$ & $\begin{array}{l}\frac{7.64}{7,34-} \\
7,89\end{array}$ & $\begin{array}{l}\frac{7.80}{7,54-} \\
8,01\end{array}$ & $\begin{array}{l}\frac{7.90}{7,71-} \\
8,12\end{array}$ & $\begin{array}{l}\frac{7.41}{7,22-} \\
7,85\end{array}$ \\
\hline Summer & $\begin{array}{l}\frac{7.37}{7,21-} \\
7,95\end{array}$ & $\begin{array}{l}\frac{7.76}{7,57-} \\
7,96\end{array}$ & $\begin{array}{l}\frac{7.97}{7,65-} \\
8,01\end{array}$ & $\begin{array}{l}\frac{8.01}{7,89-} \\
8,11\end{array}$ & $\begin{array}{l}\frac{7.32}{7,11-} \\
8,00\end{array}$ & $\begin{array}{l}\frac{8.03}{7,79-} \\
8,15\end{array}$ & $\begin{array}{l}\frac{8.09}{7,85-} \\
8,21\end{array}$ & $\begin{array}{c}\frac{8.25}{8,12-} \\
8,31\end{array}$ & $\begin{array}{c}\frac{7.57}{7,49-} \\
7,62\end{array}$ & $\begin{array}{r}7.84 \\
7,62- \\
7,96\end{array}$ \\
\hline Autumn & $\begin{array}{c}\frac{7.35}{7,19-} \\
7,56\end{array}$ & $\begin{array}{l}\frac{7.53}{7,32-} \\
7,69\end{array}$ & $\begin{array}{l}\frac{7.46}{7,28-} \\
7,70\end{array}$ & $\begin{array}{l}\frac{7.10}{6,95-} \\
7,25\end{array}$ & $\begin{array}{c}\frac{7.40}{7,29-} \\
7,49\end{array}$ & $\begin{array}{c}\frac{7.94}{7,80-} \\
8,11\end{array}$ & $\begin{array}{l}\frac{7.87}{7,76-} \\
8,09\end{array}$ & $\begin{array}{l}\frac{8.03}{7,91-}- \\
8,07\end{array}$ & $\begin{array}{c}\frac{8.12}{8,01-} \\
8,19\end{array}$ & $\begin{array}{r}\frac{7.47}{7,25-} \\
7,90\end{array}$ \\
\hline
\end{tabular}

Water conductivity in both reservoirs was on the level characteristic for natural surface waters. Autumnal results in Topiło were within range $62-69 \mu \mathrm{S} / \mathrm{cm}$ (Table 6). Dojlidy, in contrast to Topiło, stood out with lower conductivity variety during the period of researches. 
Table 6. Topiło and Dojlidy average, minimum-maximum water conductivity.

\begin{tabular}{|c|c|c|c|c|c|c|c|c|c|c|}
\hline $\begin{array}{c}\text { Conductivity } \\
{[\mu \mathrm{S} / \mathrm{cm}]}\end{array}$ & \multicolumn{5}{|c|}{ Topiło } & \multicolumn{5}{|c|}{ Dojlidy } \\
\hline Control point & 1. & 2. & 3. & 4. & 5. & 1. & 2. & 3. & 4. & 5. \\
\hline & 191 & 178 & 186 & 174 & 178 & 518 & $\underline{330}$ & $\underline{336}$ & 162 & 303 \\
\hline Spring & $\begin{array}{l}185- \\
204\end{array}$ & $\begin{array}{l}\overline{162-} \\
180\end{array}$ & $\begin{array}{l}\overline{180-} \\
191\end{array}$ & $\begin{array}{l}\overline{171-} \\
179\end{array}$ & $\begin{array}{c}\overline{169}- \\
190\end{array}$ & $\begin{array}{c}501- \\
523\end{array}$ & $\begin{array}{l}\overline{296}- \\
360\end{array}$ & $\begin{array}{l}333- \\
345\end{array}$ & $\begin{array}{l}156- \\
180\end{array}$ & $\begin{array}{l}224- \\
354\end{array}$ \\
\hline & 273 & 282 & 272 & 291 & 321 & 248 & 164 & 153 & 252 & 169 \\
\hline Summer & $\begin{array}{l}252- \\
289\end{array}$ & $\begin{array}{l}\overline{267}- \\
289\end{array}$ & $\begin{array}{c}\overline{263}- \\
278\end{array}$ & $\begin{array}{l}\overline{272}- \\
299\end{array}$ & $\begin{array}{l}\overline{290}- \\
332\end{array}$ & $\begin{array}{c}\overline{202-} \\
300\end{array}$ & $\begin{array}{l}\overline{125} \\
185\end{array}$ & $\begin{array}{l}\overline{122}- \\
160\end{array}$ & $\begin{array}{c}236- \\
268\end{array}$ & $\begin{array}{l}125- \\
205\end{array}$ \\
\hline Autumn & $\begin{array}{l}\frac{69}{62-} \\
74,5\end{array}$ & $\begin{array}{l}\frac{64}{58-} \\
102\end{array}$ & $\begin{array}{c}\frac{63}{56-} \\
69\end{array}$ & $\begin{array}{r}5 \frac{62}{59,5} \\
66\end{array}$ & $\begin{array}{l}\frac{62}{58-} \\
64,5\end{array}$ & $\begin{array}{l}\frac{268}{222-} \\
296\end{array}$ & $\begin{array}{l}\frac{160}{145-} \\
172\end{array}$ & $\begin{array}{l}\frac{157}{150-} \\
168\end{array}$ & $\begin{array}{l}\frac{159}{148}- \\
162\end{array}$ & $\begin{array}{l}\frac{173}{121-} \\
200\end{array}$ \\
\hline
\end{tabular}

During the study period, the values of analysed indicators exhibited high variability and showed characteristic patterns of seasonal changes. Carlson (1977) and Kratzer-Brezonik (1981) Trophic State Indexes were used to compare two examined reservoirs $[15,16]$. Because of low average deepness Secchi depth hasn't been measured. TSI results are presented in Figure 3.

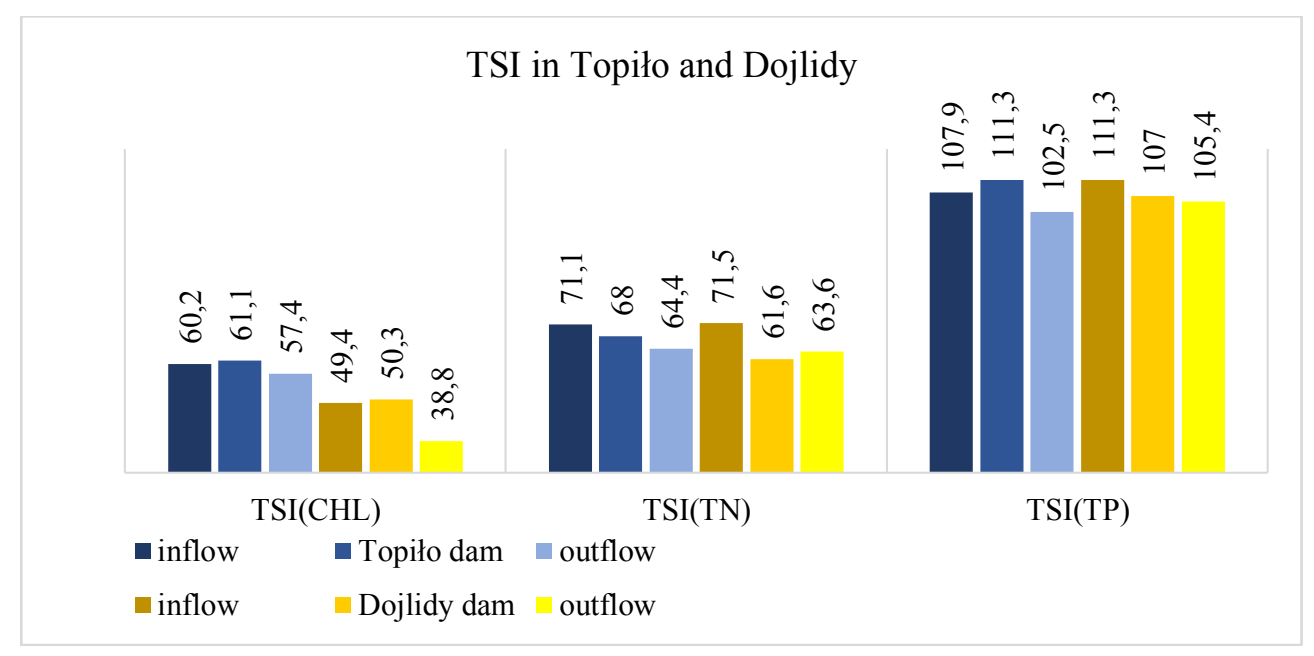

Fig. 3. Caption of the Figure 1. Below the figure.

The lowest trophic state was observed in autumn and the highest in summer. Because of the high loads of phosphorus received by the reservoirs, this element did not limit primary production. TSI values calculated based on total phosphorus were always markedly higher than calculated on chlorophyll "a" and total nitrogen. Both reservoirs demonstrated TSI indexes specific to hypertrophic lakes due to total phosphorus large amount.

Numerous studies have documented declines in water quality, habitat, and biological assemblages as the extent of agricultural land increases within catchments [17-20]. Researchers commonly report that streams draining agricultural lands support fewer species of sensitive insect and fish taxa than streams draining forested catchments [20-22]. Higher inputs of sediments, nutrients, and pesticides accompany increased agricultural land use $[23,24]$. 
Conducted researches proved that type of catchment activities has an impact on examined reservoirs water quality. Due to elevated degradation vulnerability Dojlidy reservoir was characterized by relatively high nutrients amount [25]. Moreover, researches led in 2014 by „ROLEX” Sp. z o. o. company pointed out that sediments samples from Dojlidy reservoir responded to the cultivated soil chemical composition [13].

Topiło reservoir and its tributary named Perebel River pollution level has typical natural character, which was proved by microbiological tests. They were led by i.a. Professor Edward Pierzgalski from Forest Research Institute within the expertise to determine the reasons for the Topiło water resources reduction. Researches results excepted anthropogenic impact on water quality, despite Topiło village nearness, by Topiło and Perebel high microbiological purity [26]. It should be noted that trophic state in both reservoirs was on similar, elevated level. Organic matter from wooden logs stored in the past caused lasting many years pollution.

\section{Conclusions}

1. Nutrients amount in both reservoirs was relatively high.

2. The average annual values of the TSI indexes qualify the studied reservoirs as hypertrophic.

3. Trophic state of studied reservoirs strictly dependent on the processes taking place in their vicinity.

4. Despite woody catchment area Topiło Lake water pollution was on similar level as Dojlidy with agricultural catchment area.

\section{References}

1. M. Sidoruk, A. Skwierawski, Ecol. Chem. Eng, 13(52), 337-343 (2006)

2. A. Górniak, M. Grabowska, International Rev. Hydrobiol., 83, 307-310, (1998)

3. F. Czyżyk, A. Rajmund, Ecological Engineering, 33, 29-34 (2013) (in Polish)

4. General Directorate of The State Forests, Principles of silviculture, State forests, 1114, (2012)

5. Hajnówka Forest District unpublished data

6. Google Maps (2017)

7. A. Kluczko, Elaboration for Biatystok Sport and Recreation Center, (2014) (in Polish)

8. HACH Company Loveland, Water Analysis Handbook, Colorado, (1992)

9. W. Hermanowicz, J. Dojlido, W. Dożańska, B. Koziorowski, J. Zerze, Water and wastewater physicochemical testing, (1999) (in Polish)

10. PN-C-05560-02:1986. Water and wastewater. Chlorophyll content in surface waters tests. Chlorophyll alfa measurement in planktonic algae with spectrophotometric, monochromatic method with feopigments alfa correction (in Polish)

11. Environment Minister Regulation from 21 July 2016 (Dz. U. 2016 poz. 1187) (in Polish)

12. J. Koc, J. Koc-Jurczyk, K. Solarski, Zeszyty Naukowe Polskie Towarzystwo Gleboznawcze, 121-128 (2009) (in Polish)

13. S. Rola, Bathymetric tests of Dojlidy reservoir in Biatystok, (2014) (in Polish) 
14. Z. Kajak, HYDROBIOLOGY - LYMNOLOGY. Inland waters ecosystems, (1998) (in Polish)

15. R. E. Carlson, Limnol. Oceanogr., 22(2), 361-369, (1977)

16. C. R. Kratzer, P. L. Brezonic, Water Resour. Bull., 17, (1982)

17. C. Richards, L. B. Johnson, G. E. Host, Can. J. Fish. Aquat. Sci., 53, 295-311, (1996)

18. N. E. Roth, J. D. Allan, D. L. Erickson, Landsc. Ecol., 11, 141-156, (1996)

19. R. A. Sponseller, E. F. Benfield, H. M. Valett, Freshwater Biol., 46, 1409-1424, (2001)

20. L. Wang, J. Lyons, P. Kanehl, R. Gatti, Fisheries, 22, 6-12 (1997)

21. D. Genito, W. J. Gburek, A. N. Sharpley, Freshw. Biol., 17, 109-119, (2002)

22. D. R. Lenat, J. K. Crawford, Hydrobiol., 294, 185-199, (1994)

23. C. M. Cooper, J. Environ. Qual., 22, 402-408, (1993)

24. L. B. Johnson, C. Richards, G. E. Host, J. W. Arthur, Freshw. Biol., 37, 193-208, (1997)

25. K. Oszczapińska, J. Szczykowska, Wybrane zagadnienia z zakresu ochrony środowiska i energii odnawialnej, 269-283 (2016) (in Polish).

26. E. Pierzgalski, J. Tyszka, A. Boczoń, M. Janek, M. Wróbel, A. Stolarek, K. Pachuta, P. Oględzki, M. Frąk, P. Sikorski, L. Komecka, S. Czachorowski, L. Pietrzak, M. Ksepko, Operat zarządzania wodami $i$ ochrony ekosystemów wodnych (do Planu Ochrony Białowieskiego Parku Narodowego), Forest Research Institute, 1-162, (2010) 